\title{
Efectos estigmatizantes del diagnóstico psicológico en niños
}

\author{
$\mathrm{M}^{\mathrm{a}}$ del Mar Montoya-Rodríguez ${ }^{1}$ y Francisco-Javier Molina-Cobos ${ }^{2}$ \\ ${ }^{1}$ Universidad Católica del Uruguay (Uruguay); ${ }^{2}$ Universidad de Almería (España)
}

A pesar de las ventajas conocidas de la clasificación de trastornos psicológicos, utilizar diagnósticos formales entraña el riesgo de reducir una historia de vida y modo de comportarse a una mera etiqueta diagnóstica, volviéndose una explicación tautológica que obvia la verdadera explicación del problema y añade funciones iatrogénicas y estigmatizantes. Se realiza un estudio para valorar la influencia de las etiquetas diagnósticas en la actuación de Psicólogos que evalúan la inteligencia de un grupo de niños mediante el Test de Goodenough. Diferentes dibujos de una figura humana, realizados por niños con desarrollo normalizado, fueron etiquetados como si hubieran sido dibujados por niños con retraso mental, altas capacidades y autismo. Los resultados muestran que los evaluadores, desconocedores de la falsedad diagnóstica, puntúan los dibujos diferencialmente según la etiqueta utilizada. Esto es, los resultados apoyan la influencia negativa de las etiquetas diagnósticas en las expectativas y trato hacia la persona diagnosticada.

Palabras clave: Diagnóstico psicológico, etiqueta diagnóstica, iatrogenia, estigmatización.

Stigmatizing effects of psychological diagnosis in children. Despite the known advantages of the classification of psychological disorders, using formal diagnostic could reduce a story of life and way of behaving to a mere diagnostic label, becoming a tautological explanation that ignores the true explanation of the problem and adds iatrogenic functions and stigmatizing. The present study assesses the influence of diagnostic labels in the performance of psychologists who evaluate the intelligence of a group of children. The Goodenough Test is used for instrumental reasons. The different drawings of a human figure, made by children with standardized psychological development, were labeled as if having been drawn by children with mental retardation, giftedness and normal development. The results show that evaluators, unaware of the falsity of the diagnoses, score the drawings differentially depending on the diagnostic label used. That is, the results evidence the negative influence of a diagnostic label about expectations and treatment to the person diagnosed.

Keywords: Psychological diagnosis, diagnostic label, iatrogenic, stigmatization.

Correspondencia: Francisco Javier Molina Cobos. Departamento de Psicología. Universidad de Almería. Ctra. Sacramento s/n. C.P.: 04120. Almería (España). E-mail: fjcobos@ual.es 
De igual modo que sucede con los adultos, tradicionalmente se ha considerado útil clasificar los trastornos psicológicos que tienen lugar durante la infancia y la adolescencia. Para justificar esta utilidad se ha señalado que compartir la misma nomenclatura agiliza la comunicación entre profesionales, que supone una sistematización de la información que facilita el abordaje de problemas similares así como de la investigación epidemiológica y terapéutica y, a nivel administrativo, que sirve de guía para determinar la prestación de servicios, ayudas sociales o económicas, o para delimitar las funciones específicas de diferentes profesionales (Fernández y Luciano, 1997; Lacalle, 2010; Lemos, 1995; Retolaza, 2002).

Los sistemas de clasificación DSM y CIE son los más conocidos y utilizados en el ámbito clínico de la Psicología, habiéndose convertido en el referente a la hora de llevar a cabo cualquier diagnóstico. Con el diagnóstico, un caso concreto que está siendo evaluado se asigna a una (o más) de las categorías recogidas en el sistema de clasificación correspondiente, igualándolo a otros problemas clasificados de igual forma. El diagnóstico, así dicho, significa utilizar una de las etiquetas propuestas en el sistema de clasificación. No obstante las ventajas de los sistemas de clasificación ya apuntadas, la utilización de etiquetas para clasificar el comportamiento anormal no está exenta de críticas, especialmente cuando se trata de niños. Con los diagnósticos descriptivos se corre el peligro de que lleguen a convertirse en una explicación tautológica que confunde la concreción del trastorno con el concepto explicativo; lo que conduce a interpretar las características personales, el malestar o la historia vital del paciente de acuerdo al marco de un sistema de creencias orientado a la patología (Ortiz e Ibáñez, 2011). Además, como sostienen Hayward y Bright (1997), el etiquetado es un proceso destructivo en el que la etiqueta de la enfermedad mental llega a ser más perjudicial para el individuo que el trastorno etiquetado. En relación con esto, como recoge Pokorny (1996), es habitual que la etiqueta pueda pasar de describir una conducta que está mostrando una persona a ser considerada una característica de ésta. Así, una persona a la que se le diagnostica esquizofrenia será conocida como "un esquizofrénico" y nunca volverá a ser tratado de la misma forma en su entorno social. Esto es, el etiquetado e identificación de una determinada diferencia y las expectativas creadas con respecto a esa etiqueta van a favorecer el proceso de estigmatización de la persona etiquetada (Rüsch, Angermeyer, y Corrigan, 2005). La experiencia personal de estigmatización asociada a etiquetas diagnósticas como esquizofrenia, episodio depresivo mayor o trastorno somatomorfo indiferenciado se hace evidente en los pacientes y sus familiares, llegando a afectar incluso a la adherencia al tratamiento (Acosta, Rodríguez, y Cabrera, 2013; Flores, Medina, Robles, y Páez, 2012; Lee, 2002). Como señala Pérez-Álvarez (1999), el psicodiagnóstico rotura un terreno yermo a la vez que lo rotula, en tanto que esta actuación profesional, la del psicodiagnóstico, que consiste en clasificar y nombrar, describirá la realidad dada a la vez que prescribirá su forma 
de darse. De esta manera viene a referirse el autor a que la psicología clínica, en ocasiones, trata sobre problemas que ella misma crea.

Estos efectos iatrogénicos y estigmatizantes cobran especial relevancia cuando se trata de niños o adolescentes. Son numerosos los trabajos que han mostrado los efectos negativos y estigmatizantes de las etiquetas diagnósticas en las expectativas de los maestros, los padres e incluso en los propios niños (véase la revisión realizada por Gómez, Molina, y Zaldívar, 2005). Por ejemplo, Smith, Flexer, y Sigelman (1980) examinaron mediante cuestionarios las actitudes de los directores de centros de educación primaria hacia los niños etiquetados con retraso mental, problemas de aprendizaje o niños sin diagnóstico, encontrando que los adolescentes etiquetados de alguna forma eran percibidos de manera menos positiva. En general, los adolescentes con dificultades de aprendizaje eran considerados más similares a los adolescentes con retraso mental que a los no etiquetados, especialmente en áreas relacionadas con la personalidad y la moral. Las conclusiones del estudio de Rolison y Medway (1985) apuntaban que los profesores tienden a ajustar sus niveles de expectativas según las etiquetas de educación especial, formándose mayores expectativas cuando el estudiante era etiquetado con dificultades de aprendizaje (o no tenía etiqueta) que cuando tenía la etiqueta de retraso mental. Además, se detectaron atribuciones diferenciales de factores internos y externos. Con un niño no diagnosticado, la familia era vista como un facilitador de lo que el niño podría lograr, mientras que con el niño etiquetado de retraso mental los profesores valoraban el nivel de habilidad como un factor indicativo de lo que podría llegar a alcanzar. Según esto, los maestros que trabajan con estudiantes con retraso alterarían la dificultad de la carga de trabajo para aumentar el logro académico en lugar de utilizar señales externas, como el aumento de la motivación.

Además, a la iatrogenia de la etiqueta diagnóstica se une la subjetividad del diagnóstico; esto es, la consideración de si una conducta es excesiva o deficitaria va a depender de quien emita el juicio. Como recogen Reyes-Sandoval y Acuña (2012), las maestras jóvenes de nivel socioeconómico medio que atendían a un grupo de alumnos pequeño y trabajaban en escuela privada juzgaban ciertas conductas como anormales en mayor medida que sus compañeros docentes de características opuestas.

Son escasos los trabajos que analizan los efectos negativos del uso de etiquetas diagnósticas de corte nosológico en la actividad de los expertos en el comportamiento infantil, como estudiantes y titulados de Psicología y Psiquiatría. En esta línea, Eikeseth y Lovaas (1992) encontraron que estudiantes de Psicología proporcionaban más elogios y menos correcciones verbales ante las respuestas incorrectas cuando los niños eran etiquetados como autistas, por comparación con niños etiquetados como normales; no encontrando diferencias en las consecuencias proporcionadas ante respuestas correctas de unos y otros. En una reciente replicación de este trabajo, Barbero, Molina-Cobos, Eisenbeck, y López (2016) obtienen resultados similares. Por su parte, Dryer, Kiernan, y Tyson (2006) apuntaban que la presencia de la etiqueta de TDAH en la descripción de un 
niño que mostraba comportamientos de falta de atención, impulsividad e hiperactividad cambiaba las creencias de profesionales de la salud (psicólogos, terapeutas ocupacionales, trabajadores sociales, nutricionistas, profesores de primaria y educadores especiales) sobre los factores causales de estos comportamientos, pero no sus creencias sobre la mejor manera de abordarlos o manejarlos. Concretamente, con la presencia de la etiqueta diagnóstica los profesionales eran más propensos a atribuir la causa de los comportamientos a factores neurológicos o genéticos, mientras que sin la etiqueta de diagnóstico del TDAH estos comportamientos se atribuían a factores en el entorno del niño y al comportamiento de los padres.

Dada la importancia de examinar las repercusiones de la etiqueta diagnóstica en la actuación de titulados en Psicología, quienes juegan un papel crucial en el tratamiento de niños con dificultades en el desarrollo, el presente estudio se llevó a cabo con graduados en Psicología que debían evaluar dibujos realizados por niños con un desarrollo normalizado a quienes, de cara a la realización del estudio, se les habían asignado tres etiquetas diagnósticas diferentes: retraso mental, altas capacidades y desarrollo normal. A partir de la evaluación de los dibujos los participantes debían atribuir a los niños un nivel de desarrollo intelectual. Se perseguía analizar si los diferentes diagnósticos de un mismo dibujo daban como resultado diferentes puntuaciones en las evaluaciones.

\section{MÉTODO}

\section{Participantes}

Dieciocho titulados en Psicología (12 mujeres y 6 hombres) participaron como evaluadores. En el momento de la realización del estudio todos ellos cursaban estudios de postgrado. Su colaboración fue voluntaria y no recibieron ninguna contraprestación a cambio. Ninguno de los participantes estaba familiarizado con el instrumento de evaluación que iba a ser utilizado.

\section{Instrumentos}

Escala de Inteligencia de F. Goodenough (Goodenough, 1926). Es una prueba proyectiva de inteligencia no verbal. Esta prueba, también conocida como test de la figura humana, evalúa la inteligencia general mediante el análisis del dibujo de la figura de un hombre para el que se requiere poner de manifiesto funciones tales como sentido espacial, abstracción o coordinación viso-manual. El análisis del dibujo se limita a computar el número de detalles que incorpora la figura, siguiendo unos criterios establecidos previamente en una guía de 51 ítems descritos en el correspondiente manual de puntuación. El sistema de puntuación consiste en sumar un punto por la presencia en el dibujo de cada uno de los elementos establecidos. Si un elemento no está presente se puntúa 0 . Una vez 
obtenida la puntuación total, se consulta la edad mental en la tabla de baremación correspondiente. Con estos datos se calcula el CI.

Para la evaluación mediante el test Goodenough se utilizaron 17 dibujos realizados por niños de 3-4 años de edad, con un desarrollo normalizado, a los que su profesora de educación infantil pidió que dibujasen en una hoja de papel la figura de un hombre de "cuerpo entero". En todo momento se garantizó el anonimato de los niños, evitando que pusieran su nombre o cualquier otro dato identificativo.

\section{Diseño}

Con los participantes (evaluadores) se conformaron tres grupos de seis componentes cada uno. Quince dibujos fueron distribuidos en tres carpetas, con cinco dibujos cada una. Los dos dibujos restantes fueron utilizados en una fase previa de entrenamiento en el uso del manual de corrección del test. Las carpetas eran identificadas con tres etiquetas diferentes: altas capacidades, retraso mental y desarrollo normal. Como se resume en la tabla 1, la etiqueta que se colocaba a las carpetas variaba según el grupo de evaluadores. De esta forma todos los participantes valoraban los quince dibujos y todos los dibujos fueron identificados con las tres etiquetas, evitando así que un evaluador encontrara el mismo dibujo etiquetado de formas diferentes.

Tabla 1. Distribución de las carpetas (dibujos) según etiquetas y evaluadores

\begin{tabular}{cccc}
\hline & \multicolumn{3}{c}{ Etiquetas } \\
\hline Evaluadores & Altas capacidades & Retraso mental & Desarrollo Normal \\
\hline Grupo 1 & Carpeta1 & Carpeta2 & Carpeta3 \\
\hline Grupo 2 & Carpeta3 & Carpeta1 & Carpeta2 \\
\hline Grupo 3 & Carpeta2 & Carpeta3 & Carpeta1 \\
\hline
\end{tabular}

Se planteó un diseño unifactorial con una variable independiente con tres niveles (altas capacidades, retraso mental y desarrollo normal), lo que dio lugar a tres condiciones experimentales según la etiqueta diagnóstica. La variable medida era la puntuación total otorgada a los dibujos.

\section{Procedimiento}

Para la obtención de los dibujos se contactó con una maestra de educación infantil a quien se solicitó su colaboración a tal efecto. Se le dijo que entregara a los niños una hoja de papel y les pidiera que dibujaran una persona "de cuerpo entero" lo mejor que pudieran. Una vez obtenidos los dibujos se procedió a solicitar la colaboración de los estudiantes de postgrado que harían las veces de evaluadores. Para ello se les dijo que se solicitaba su participación, como titulados en Psicología, para la realización de una investigación en la que se estaban comparando diferentes pruebas de evaluación y/o diagnóstico con el objetivo de comprobar si, en general, son tan diferentes como a priori se suele pensar o, por el contrario, podrían ser similares en sus resultados. A ellos les había 
correspondido llevar a cabo la parte referida a la evaluación con el Test Goodenough. Se les describió la prueba y sus características principales y se les dijo que su tarea se limitaba a valorar y puntuar los dibujos. Se insistió en que cada uno de ellos, de manera individual, debería valorar varios dibujos, sin que pudieran compartir información alguna con el resto de participantes.

Los dibujos fueron tratados digitalmente y almacenados en línea en la plataforma Google docs ${ }^{\circledR}$. De esta manera los participantes podían consultar la guía de corrección del test y todo el material necesario desde su propio ordenador gracias al enlace que se les facilitaba a través del correo electrónico. A través de este medio se les informó de la disponibilidad de los dibujos y de las plantillas de corrección individualizadas y recibían las instrucciones pertinentes en cada momento.

En primer lugar se facilitaron a los participantes dos dibujos de prueba, sin ninguna etiqueta diagnóstica, para que se familiarizasen con el test y los criterios de corrección. Una semana más tarde tuvieron disponible el material de evaluación. Se les hizo saber que los dibujos estaban agrupados según diferentes diagnósticos de los niños que los habían realizado y que estos diagnósticos venían dados por otros profesionales. En la parte superior de cada dibujo aparecía un código de identificación compuesto por un número y el diagnóstico del niño que lo realizaba (por ejemplo, Retraso Mental-1, Retraso Mental-2, etc.). Las plantillas de corrección aparecían identificadas de la misma forma. Dispusieron de 15 días para realizar todas las evaluaciones. Se consideró que, dado el número de dibujos a evaluar, las características de la tarea y la poca experiencia de los participantes en el uso del protocolo, dos semanas podría ser tiempo suficiente para que los participantes pudieran llevar a cabo su cometido convenientemente (esto es, de forma equivalente a cómo lo podría hacer un profesional que utilizara esta prueba diagnóstica). Pasado este tiempo debían entregar las plantillas de corrección de los diferentes dibujos directamente a los experimentadores. En cada plantilla aparecía la puntuación de los diferentes ítems y el sumatorio total. En ningún caso se demoró la entrega del material debidamente cumplimentado.

\section{RESULTADOS}

Las puntuaciones medias y desviaciones típicas de las tres condiciones (altas capacidades, retraso mental y desarrollo normal) se presentan en la tabla 2. Los datos aparecen organizados según los grupos de dibujos (carpetas). Así, se analizan los resultados para cada carpeta de dibujos, cada una de las cuales contiene los mismos dibujos en las tres condiciones. Los dibujos de las tres carpetas obtienen las puntuaciones más bajas cuando están identificados con la etiqueta altas capacidades. Además, la carpeta 1 y la carpeta 3 reciben una menor puntuación con la etiqueta retraso mental que con desarrollo normal (Figura1). 
Tabla 2. Medias $(M)$ y desviaciones típicas $(D T)$ de las tres condiciones en cada carpeta

\begin{tabular}{lcccccc}
\hline & \multicolumn{2}{c}{ Altas Capacidades } & \multicolumn{2}{c}{ Retraso Mental } & \multicolumn{2}{c}{ Desarrollo Normal } \\
\hline & $M$ & $D T$ & $M$ & $D T$ & $M$ & $D T$ \\
\hline Carpeta 1 & $23.63^{\mathrm{a}}$ & 3.49 & $24.00^{\mathrm{c}}$ & 3.17 & $24.77^{\mathrm{b}}$ & 1.59 \\
\hline Carpeta 2 & $23.70^{\mathrm{b}}$ & 3.69 & $32.07^{\mathrm{a}}$ & 4.76 & $29.13^{\mathrm{c}}$ & 4.13 \\
\hline Carpeta 3 & $26.60^{\mathrm{c}}$ & 5.73 & $27.03^{\mathrm{b}}$ & 4.59 & $30.23^{\mathrm{a}}$ & 5.97 \\
\hline Media etiqueta & 24.64 & 4.34 & 27.7 & 5.22 & 28.04 & 4.67 \\
\hline Nota: a=Evaluadores 1; b=Evaluadores 2; c=Evaluadores 3 & &
\end{tabular}

Para el análisis estadístico de estos resultados se ha aplicado la prueba de análisis de la varianza para un factor (ANOVA) en cada una de las carpetas, con un valor crítico asociado de $\alpha=.05$. Este análisis muestra que existen diferencias estadísticamente significativas únicamente entre las medias de la carpeta $2, F_{(2,12)}=5.06, p=.02$, concretamente entre los diagnósticos altas capacidades y retraso mental $(p=.02)$.

De manera global, atendiendo a la puntuación total de las etiquetas, se tiene que la etiqueta con menor puntuación es altas capacidades, seguida por la etiqueta retraso mental y, con una puntuación media global que difiere escasamente, por desarrollo normal. En este caso, el análisis estadístico realizado ha sido el modelo univariado ANOVA para medidas repetidas, arrojando que existen diferencias estadísticamente significativas entre las etiquetas, $F_{(2,28)}=7.33, p=.003$, concretamente entre las etiquetas altas capacidades y desarrollo normal $(p=.001)$.

Figura 1. Puntuaciones medias de altas capacidades (negro), retraso mental (blanco) y desarrollo normal (gris) en los dibujos de las tres carpetas y la puntuación media total

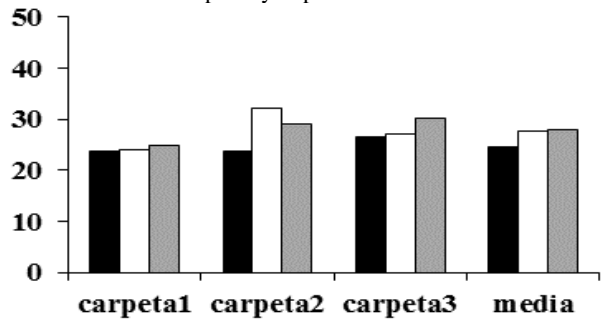

El primer grupo de evaluadores otorga la puntuación más baja a los dibujos con la etiqueta altas capacidades, obteniendo la puntuación más alta los etiquetados como retraso mental (Figura 2). Lo mismo sucede con el segundo grupo de evaluadores. Por el contrario, el tercer grupo da la puntuación más baja a los dibujos con la etiqueta retraso mental, seguidos de los etiquetados como altas capacidades y desarrollo normal. En términos generales, como ya se ha dicho, la etiqueta altas capacidades obtiene la puntuación más baja si se atiende a los grupos de evaluadores.

En la tabla 3 se recogen las puntuaciones dadas a todos los dibujos por los diferentes evaluadores en cada una de las condiciones. Se observa que 9 de los 15 dibujos obtienen las puntuaciones medias más bajas cuando son evaluados con la etiqueta altas 
capacidades y 4 cuando son etiquetados con retraso mental (la puntuación media del dibujo número 2 coincide en ambas etiquetas). Solo uno de los 15 dibujos alcanza una puntuación media más baja con la etiqueta desarrollo normal. Por otro lado, 6 de los 15 dibujos obtienen la valoración más alta con la etiqueta retraso mental, y 8 cuando están etiquetados con desarrollo normal. El dibujo número 3 obtiene la máxima puntuación media tanto con la etiqueta altas capacidades como con la etiqueta retraso mental.

Figura 2. Puntuaciones medias de los diferentes evaluadores a las etiquetas altas capacidades (negro), retraso mental (blanco) y desarrollo normal (gris)

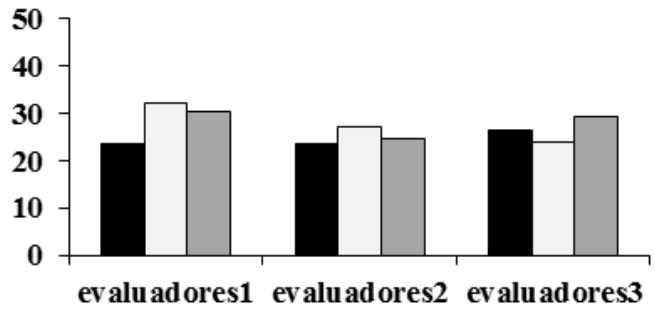

Tabla 3. Puntuaciones directas y puntuaciones medias de los dibujos en las diferentes condiciones (etiquetas)

\begin{tabular}{|c|c|c|c|c|c|c|c|c|c|c|c|c|c|c|}
\hline D1 & D2 & D3 & $\mathrm{D} 4$ & D5 & D6 & D7 & D8 & D9 & D10 & D11 & D12 & D13 & D14 & D15 \\
\hline 17 & 23 & 31 & 24 & 29 & 20 & 22 & 15 & 22 & 17 & 16 & 27 & 31 & 24 & 30 \\
\hline 21 & 28 & 29 & 26 & 28 & 25 & 19 & 16 & 27 & 20 & 16 & 24 & 27 & 21 & 25 \\
\hline 24 & 20 & 28 & 26 & 26 & 24 & 30 & 34 & 38 & 23 & 19 & 41 & 40 & 32 & 37 \\
\hline 17 & 16 & 29 & 24 & 28 & 20 & 19 & 23 & 24 & 22 & 18 & 26 & 30 & 29 & 30 \\
\hline 18 & 11 & 24 & 18 & 22 & 20 & 22 & 33 & 33 & 21 & 17 & 29 & 33 & 30 & 29 \\
\hline$<26$ & 24 & 29 & 20 & 23 & 17 & 23 & 27 & 34 & 21 & 16 & 24 & 30 & 21 & 26 \\
\hline $\bar{X} \quad 20.50$ & 20.33 & 28.33 & 23.00 & 26.00 & 21.00 & 22.50 & 24.67 & 29.67 & 20.67 & 17.00 & 28.50 & 31.83 & 26.17 & 29.50 \\
\hline-24 & 17 & 25 & 16 & 25 & 27 & 32 & 38 & 34 & 31 & 23 & 26 & 30 & 30 & 24 \\
\hline 23 & 17 & 25 & 18 & 20 & 29 & 29 & 35 & 40 & 26 & 20 & 27 & 32 & 31 & 26 \\
\hline 28 & 22 & 34 & 28 & 31 & 20 & 28 & 28 & 28 & 22 & 19 & 26 & 35 & 29 & 30 \\
\hline 24 & 22 & 29 & 23 & 22 & 28 & 37 & 41 & 39 & 27 & 18 & 24 & 34 & 34 & 26 \\
\hline 27 & 18 & 27 & 22 & 25 & 32 & 35 & 42 & 39 & 31 & 15 & 35 & 28 & 17 & 21 \\
\hline 24 & 26 & 30 & 22 & 26 & 26 & 33 & 39 & 38 & 28 & 24 & 31 & 33 & 35 & 28 \\
\hline $\bar{X} \underline{25.00}$ & 20.33 & $\underline{28.33}$ & 21.50 & 24.83 & $\underline{27.00}$ & $\underline{32.33}$ & 37.17 & $\underline{36.33}$ & $\underline{27.50}$ & 19.83 & 28.17 & 32.00 & 29.33 & 25.83 \\
\hline $\bar{\pi} 24$ & 26 & 24 & 28 & 25 & 24 & 28 & 32 & 31 & 24 & 23 & 31 & 33 & 35 & 30 \\
\hline 25 & 23 & 19 & 28 & 20 & 18 & 30 & 24 & 30 & 18 & 16 & 29 & 38 & 23 & 32 \\
\hline 20 & 25 & 14 & 25 & 18 & 34 & 40 & 37 & 38 & 34 & 13 & 25 & 27 & 24 & 22 \\
\hline 27 & 19 & 34 & 27 & 36 & 24 & 28 & 28 & 37 & 21 & 22 & 39 & 40 & 32 & 38 \\
\hline 31 & 26 & 33 & 28 & 34 & 21 & 28 & 37 & 33 & 25 & 21 & 35 & 37 & 35 & 33 \\
\hline$\subseteq 22$ & 19 & 16 & 23 & 24 & 26 & 29 & 31 & 35 & 29 & 25 & 37 & 38 & 35 & 39 \\
\hline $\bar{X} 24.83$ & $\underline{23.00}$ & 23.33 & 26.50 & $\underline{26.17}$ & 24.50 & 30.50 & 31.50 & 34.00 & 25.17 & $\underline{20.00}$ & 32.67 & 35.50 & 30.67 & $\underline{32.33}$ \\
\hline
\end{tabular}

Marcadas en color gris aparecen las puntuaciones medias más bajas para cada dibujo, y subrayadas las más altas.

\section{DISCUSIÓN Y CONCLUSIONES}

Se ha planteado como objetivo primordial de este estudio analizar si las etiquetas diagnósticas asignadas a unos niños influyen en la valoración que de ellos hacen unos 
expertos. En este caso estudiantes de postgrado de Psicología a los que se les pide que valoren el nivel de inteligencia de un grupo de niños de educación infantil a través de la evaluación de sus dibujos. Los dibujos, realizados por niños del mismo nivel y grupo escolar con un desarrollo normal y sin marcadas diferencias intelectuales entre ellos, son presentados a los expertos como si hubieran sido hechos por niños con altas capacidades, con retraso mental y con un desarrollo normal. Esto es, los dibujos utilizados no debieran diferir sustancialmente si no es en cómo son etiquetados. Así, cualquier diferencia sustancial en su valoración debería entenderse mediada por la etiqueta diagnóstica asociada.

Para la evaluación de los dibujos se hace uso de una prueba que ha sido puesta en entredicho como herramienta útil de evaluación (p.ej., Ter Laack, de Goede, Aleva, y van Rijswijk, 2005). Cabe hacer hincapié, no obstante, en que ésta es una cuestión menor, en tanto que no es propósito de este estudio ni valorar la fiabilidad o validez de la prueba ni evaluar capacidad o habilidad alguna en personas concretas. Su utilización es meramente instrumental y se opta por la utilización de esta prueba por la facilidad de su uso y la disponibilidad de un protocolo estructurado que, por su minuciosidad, sirve de guía de cómo deben ser puntuados diferentes aspectos de los dibujos valorados.

Dado que el interés último del estudio radica en conocer el efecto que pueden tener las etiquetas diagnósticas en la labor de los profesionales, se ha contado con la participación de estudiantes de postgrado de Psicología. En tanto que ninguno había utilizado previamente el material de evaluación, se les ha facilitado la guía de corrección de la prueba y dos dibujos para que practicaran, llegando a tener todos una experiencia similar en su manejo.

En cuanto a los resultados obtenidos, en síntesis, muestran que los dibujos son puntuados diferencialmente dependiendo de la etiqueta diagnóstica mostrada. Más concretamente, cuando los dibujos son identificados con la etiqueta altas capacidades son valorados por los evaluadores con una puntuación menor que cuando se identifican con otras etiquetas. Así ocurre con 9 de los 15 dibujos. En otros casos (4 de los 15 dibujos) reciben la menor puntuación cuando llevan la etiqueta retraso mental. Esto así, y dado que la única variabilidad radica en las diferentes etiquetas diagnósticas utilizadas para la identificación de los dibujos, podría concluirse que las diferencias observadas en los resultados serían atribuibles a la influencia diferencial del diagnóstico en los evaluadores.

Atendiendo a las puntuaciones dadas a cada uno de los dibujos se puede observar que, en algunos casos, las puntuaciones varían muy poco de unas etiquetas a otras (y así, de un evaluador a otro). Esto debería ser así con todos los dibujos si las etiquetas diagnósticas no estuvieran influyendo en la valoración que se hace de ellos. Esto es, el protocolo de la prueba de evaluación recoge los criterios de forma suficientemente sistematizada como para que dos evaluadores diferentes puntúen de forma similar el mismo dibujo. Que esto ocurra con unos dibujos y no con otros apuntaría a que la influencia de la etiqueta diagnóstica se hace más relevante cuando los dibujos son más complejos, en el sentido de que tienen más 
elementos criterio a valorar o estos no están suficientemente claros, y el evaluador debe interpretar esa posible ambigüedad (Barbero, Molina-Cobos, Eisenbeck, y López, 2016). Por contra, habrá mayor coincidencia entre evaluadores si un dibujo contiene pocos elementos que deban necesitar del criterio del evaluador, por estar claramente presentes (o ausentes), estando la evaluación, así, libre de interpretaciones diferentes por parte de evaluadores diferentes.

En la misma dirección que estudios anteriores (Eikeseth y Lovaas, 1992), cabía esperar que, de tener las etiquetas diagnósticas algún efecto diferencial en la actuación de los participantes, los dibujos con la etiqueta retraso mental deberían obtener las puntuaciones más altas. Los participantes del estudio de Eikeseth y Lovaas, estudiantes de Psicología, alababan más y corregían menos las respuestas incorrectas de los niños etiquetados como autistas. De forma similar, los resultados de este estudio muestran que esto es así con 6 de los 15 dibujos, que son valorados con la puntuación más alta cuando están etiquetados con retraso mental, muy por encima de lo que ocurre con la etiqueta altas capacidades. Eso sí, siendo llamativa, esta diferencia solo resulta estadísticamente significativa con los dibujos de la carpeta número 2. Dicho de otra forma, los evaluadores se muestran más exigentes cuando el dibujo está etiquetado con altas capacidades (un mayor número de dibujos es peor valorado con esta etiqueta y ninguno obtiene la puntuación más alta con ella) y, quizá, más condescencientes cuando el dibujo está etiquetado con retraso mental. Este resultado podría mostrar que los evaluadores se rigen por un criterio más exigente cuando deben evaluar un dibujo realizado por un niño de altas capacidades, quizá porque esperan un alumno con mejores habilidades de razonamiento, amplios conocimientos y un mejor desarrollo. Esto mismo serviría para explicar la diferencia, también con significación estadística, entre altas capacidades y desarrollo normal. Llevado a un contexto real, tales creencias tradicionales sobre lo que significa ser superdotado y talentoso van a afectar a las respuestas educativas adecuadas que necesitan niños con alto rendimiento (Moon y Brighton, 2008). Como recogen Ramiro, Navarro, Menacho, López y García (2016), en la literatura especializada ha sido tratada la impopularidad de los niños superdotados, afirmándose en algunos casos la existencia del estigma de la superdotación.

A partir de estos resultados, que señalan diferencias palpables en varias direcciones ( si bien no notas con significación estadística), y como ya han apuntado estudios anteriores, es conveniente poner de relieve los efectos negativos de un diagnóstico formal de corte nosológico en la población infantil. En suma, este estudio sería un ejemplo más del efecto perjudicial que puede ejercer una etiqueta diagnóstica sobre las expectativas y el trato diferencial hacia la persona diagnosticada y, en este caso, las consecuencias negativas en cuanto a la evaluación/valoración de la capacidad intelectual medida mediante una prueba diagnóstica concreta. Cabe mencionar, no obstante, que la muestra de evaluadores cuenta con poca experiencia en el trato con niños diagnosticados de una u otra forma, por lo que las diferencias observadas según las etiquetas deberán ser atribuidas a actitudes 
preconcebidas. En ese sentido, resultaría de interés llevar a cabo un estudio similar a este con profesionales experimentados en el trabajo con niños con diferentes problemas psicológicos. Cabría plantear finalmente que si bien la metodología presentada es sencilla, aún podría abordarse un diseño que permitiera que todos los evaluadores valoraran los mismos dibujos etiquetados de diferente forma. Esto es, poder obtener medidas intrasujeto con varias replicaciones dentro del mismo estudio.

\section{REFERENCIAS}

Acosta, F., Rodríguez, L., y Cabrera, B. (2013). Beliefs about depression and its treatments: Associated variables and the influence of beliefs on adherence to treatment. Revista de Psiquiatría y Salud Mental, 6(2), 86-92.

Barbero, A., Molina Cobos, F.J., Eisenbeck, N., y López, J.C. (2016). Efecto diferencial de la etiqueta diagnóstica autista en la intervención con niños. International Journal of Psychology and Psychological Therapy, 3, 281-293.

Dryer, R., Kiernan, M.J., y Tyson, G.A. (2006). The effects of diagnostic labelling on the implicit theories of Attention Deficit/Hyperactivity Disorder held by health professionals. Behaviour Change, 23(3), 177-185.

Gómez, I., Molina, A., y Zaldívar, F. (2005). Una revisión sobre los posibles efectos iatrogénicos y estigmatizantes de la clasificación diagnóstica en el ámbito infantil y alternativas de corte funcional. Revista Mexicana de Psicología, 22(1), 69-78.

Goodenough, F.L. (1926). Measurement of intelligence by drawings. New York: Harcourt, Brace.

Eikeseth, S., y Lovaas, O.I. (1992). The autistic label and its potentially detrimental effect on the child'streatment. Journal of Behavior Therapy and Experimental Psychiatry, 23(3), 151157.

Fernández, A., y Luciano, M.C. (1997). Clasificación de las alteraciones psicológicas en la infancia. En M.C. Luciano (Ed.) Manual de Psicología Clínica. Infancia y adolescencia (pp.71-86). Valencia: Promolibro.

Flores, S., Medina, R., Robles, R., y Páez, F. (2012). Estigma y apego al tratamiento psiquiátrico en los trastornos mentales severos y persistentes. Revista Latinoamericana de Psiquiatría, 11(3), 82-86.

Hayward, P., y Bright, H.P. (1997). Stigma and mental illness: A review and critique. Journal of Mental Health, 6(4), 1-11.

Lacalle, M. (2010). Escalas DSM del CBCL y YSR en niños y adolescentes que acuden a consulta en servicios de salud mental. (Tesis de maestría, Universidad Autónoma de Barcelona). Recuperado de http://hdl.handle.net/10803/5475

Lee, S. (2002). Socio-cultural and global health perspectives for the development of future psychiatric diagnostic systems. Psychopathology, 35, 152.

Lemos-Giráldez, S. (1995). Clasificación y diagnóstico en psicopatología. En A. Belloch, B. Sandín y F. Ramos (Eds.). Manual de psicopatología (Vol. 1) (pp. 127-161). Madrid: McGrawHill.

Moon, T. R., y Brighton, C. M (2008). Primary Teachers' Conceptions of Giftedness. Journal for the Education of the Gifted, 31(4), 447-480.

Ortiz A., e Ibáñez, V. (2011). Iatrogenia y prevención cuaternaria en salud mental. Revista Española de Salud Pública, 85(6), 513-523.

Pérez-Álvarez, M. (1999). Psicología clínica e iatrogénesis. En J. Buendía (Ed.). Psicología Clínica. Perspectivas actuales (pp. 33-50). Madrid: Pirámide. 
Pokorny, A. (1996). Problems of Psychiatric classification. International Journal of Neuropsychiatry, 1(2), 161-167.

Ramiro, P., Navarro, J.I., Menacho, I., López, M.M., y García, M.A. (2016). Bienestar psicológico en personas con alta capacidad intelectual. European Journal of Education and Psychology, 9, $72-78$.

Retolaza, A. (2002) La psicopatología insustancial en la era del DSM IV y la CIE-10. Revista de la Asociación Española de Neuropsiquiatría, 82, 67-73.

Reyes Sandoval, A.M., y Acuña, L. (2012). Juicios de maestros sobre las conductas características del Trastorno por Déficit de Atención e Hiperactividad. Revista Latinoamericana de Psicología, 44(3), 65-82.

Rolison, M.A., y Medway, F.J. (1985). Teachers expectations and attributions for student achievement: Effects of label, performance pattern, and special education intervention. American Education Research Journal, 22, 561- 573.

Rüsch, N., Angermeyer, M.C., y Corrigan, P. (2005). Mental illness stigma: Concepts, consequences and initiatives to reduce stigma. European Psychiatry, 20, 529-539

Smith, T.E., Flexer, R.W., y Sigelman, C.K. (1980). Attitudes of secondary principals toward the learning disabled, the mentally retarded and workstudy programs. Journal of Learning Disabilities, 13, 62-64.

Ter Laak, J., de Goede, M., Aleva, A., y van Rijswijk, P. (2005). The Draw-A-Person Test: An indicator of children's cognitive and socioemotional adaptation? Journal of Genetic Psychology, 166(1), 77-93.

Recibido: 28 de septiembre de 2016 Recepción Modificaciones: 24 de enero de 2017 Aceptado: 6 de febrero de 2017 